\title{
Auger Electron Spectroscopy of Carbon Diffusion Profiles in Low Temperature Carburized Stainless Steels
}

\author{
Wayne Jennings, Amir Avishai, Ben Cowen, Harold Kahn, Frank Ernst, Arthur H. Heuer \\ Swagelok Center for Surface Analysis of Materials, Case Western Reserve University, Cleveland, \\ $\mathrm{OH}, 44106$
}

AISI 316L and $254 \mathrm{SMO}$, grades of austenitic stainless steels were carburized by a lowtemperature gas-phase process. Using calibrated scanning Auger microprobe analysis of crosssectional specimens under dynamic sputtering, we determined the concentration-depth profile of carbon.

Characterizing the hardened steels using metallographic methods (fig 1a) or through their hardness profiles (fig 1b) provides important information as to their change in properties following this relatively novel process [1], yet it does not provide any compositional information that allows understanding of the diffusion behavior of carbon [2]. GDOES (glow discharge optical emission spectroscopy) measurements, are not very reliable for measuring such deep profiles. Using EDS/WDS, carbon buildup as a function of measuring time makes measurement of accurate data very challenging, and for other steels in which very thin cases are formed (a few microns) gives very limited data and an averaged composition due to the electron interaction volume.

Auger characterization of these materials has the advantage of providing higher spatial resolution information and hence a more detailed compositional profile. However, due to the nature of the technique, long acquisition times, and surface migration of contaminating carbon, complicate the measurement process and lead to the need for continuous ion sputtering during acquisition of Auger spectra. This process in turn leads to the further complication of differential sputtering effects which alters the surface composition of the material.

This study details the corrections to the Auger relative sensitivity factors needed to achieve accurate characterization of these materials under dynamic sputtering. Fundamental analytical analysis of carbon surface diffusion supports the appropriateness of the method. Using simplified Monte Carlo simulations applied for a monolayer of the alloy, it can be shown that the dynamic sputtering results in alteration of the surface composition which can be calculated based on the differential sputtering coefficients. Still, a steady state is reached that reflects the original bulk composition altered by the differential sputtering (fig $2 \mathrm{a}$ ). A complete analytical solution to the effect of differential sputtering is complicated by the variation of Auger sampling depth due to variations in the inelastic mean free paths of these electrons over the spectrum. This overcomes the difficulty of solving analytically the corrected sensitivity factors over the few atomic layers that are actually measured. We have addressed this issue by empirically measuring the carbon sensitivity factor using standards under similar conditions; these were applied to the as-measured values (Fig 2b) to obtain the corrected compositional profiles (fig 1b). 
The depth profile measured is concave and very different from the error-function-like shape expected for concentration independent diffusion. The measurements indicate carbon levels up to 15 at.\% in solid solution and a total case depth of $30 \mu \mathrm{m}$ (fig 1b, 2b) [2]. The carbon concentration levels measured were confirmed as well with LEAP (local electrode atom probe) analysis carried out on samples prepared from the case surface [3].

\section{References}

[1] N. Agarwal, Acta Mater., 55 (2007) 5572.

[2] F. Ernst et al., Metall. Mater. Trans. A,Vol. 40A (2009) 1768.

[3] A. Avishai, et al., Microsc. Microanal., 13, Suppl 2, (2007) 1094D.

a.

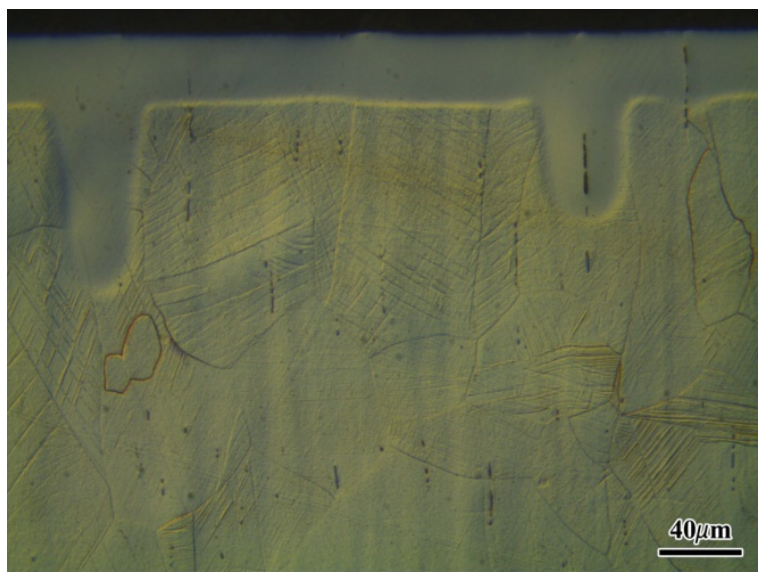

b.

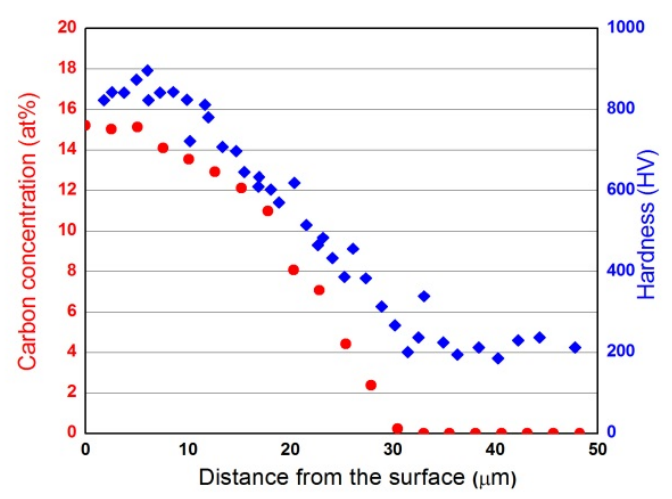

Figure 1. a, Cross-sectional optical micrograph of a carburized 316L stainless steel sample that had been electropolished prior to carburization and metallographically polished and etched to reveal the hardened case along the top of the image. b) Vickers hardness (diamond) and carbon concentration (circles) depth profile determined by calibrated scanning Auger microprobe analysis of the $316 \mathrm{~L}$ metallographic cross-section under dynamic sputtering conditions.

a.

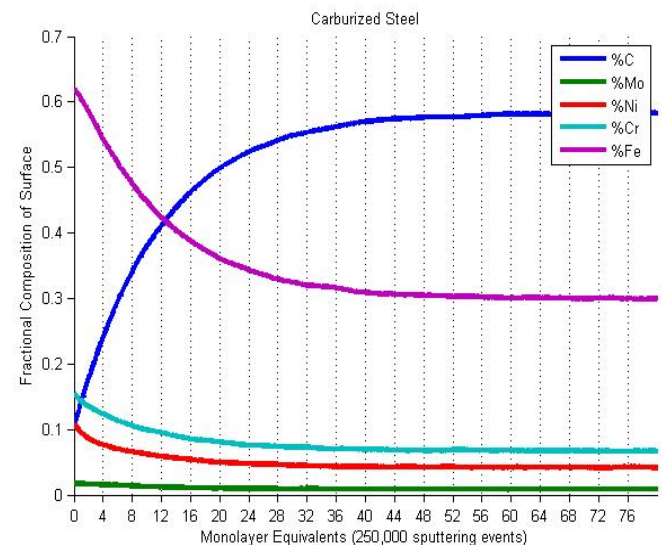

Figure 2. a. Monte Carlo type simulation of surface composition alteration during sputtering of a $10 \%$ carbon stainless steel. Note enhanced carbon on the surface after sputtering. b). Uncalibrated carbon concentrations depth profiles as determined by scanning Auger microprobe analysis of a metallographic cross-sections under dynamic sputtering conditions. 